years old; $75 \%$ taking $\geq 3$ capsules per dose). $88.57 \%$ vs. $85.71 \%$ of patients took their pills in cycles of 5 days followed by 23 days without treatment.

Satisfaction pre- and post-intervention was related to: the number of capsules prescribed per dose $(4.43 \pm 1.60 \mathrm{vs}$. $4.96 \pm 0.84)$, the possibility of taking their treatment everywhere $(5.17 \pm 0.92$ vs. $5.32 \pm 0.82)$, and the convenience of the chemotherapeutic regime $(5.06 \pm 0.94$ vs. $5.07 \pm 1.05)$.

The usefulness of the pharmaceutical attention, the pillbox and the leaflet were valuated as $5.46 \pm 0.58,5.39 \pm 0.69$ and $5.68 \pm 0.48$, respectively. Global satisfaction with pharmaceutical attention was $5.79 \pm 0.42$.

Conclusions In this study, information provided by hospital pharmacist and the use of pillboxes improved satisfaction in patients treated with temozolomide.

No conflict of interest.

\section{OHP-026 ECONOMIC EVALUATION OF ANTIFUNGAL DRUGS IN AN INTENSIVE CARE UNIT}

doi:10.1136/ejhpharm-2013-000276.400

${ }^{1} \mathrm{~F}$ Cattel, ${ }^{2} \mathrm{M}$ Chiumente, ${ }^{3} \mathrm{~F}$ Ancona, ${ }^{3} \mathrm{~A}$ Miliacca, ${ }^{4} \mathrm{FG}$ De Rosa, 'M Scaldaferri, 'E Sciorsci, 'F Re, 'S Stecca. 'A.O.U. Città della Salute e della Scienza, Hospital Pharmacy, Turin, Italy; ${ }^{2}$ University of Turin, School of hospital pharmacy, Turin, Italy; ${ }^{3}$ PricewaterhouseCooper Advisory s.p.a., Pharmaceutical \& Life Sciences, Turin, Italy; ${ }^{4}$ C.O. Amedeo di Savoia-Birago di Vische, Amedeo di Savoia Hospital, Turin, Italy

Background Anidulafungin is a semisynthetic echinocandin, mainly used in invasive Candida infections in non-neutropenic patients, with a daily dose cost lower than other antifungal drugs used in candidiasis treatment.

Purpose To prepare a mathematical model, able to produce an estimate of the savings that could be realised using anidulafungin instead of the other antifungals.

Materials and Methods A pilot study was carried out at Turin hospital 'Città della Salute e della Scienza', involving two Intensive Care Units (ICUs), which are the major consumers of echinocandins.

In these two ICUs:

- Data concerning consumption, prices and 2010 rebates for various antimycotics were collected;

- The medical records of 174 patients, admitted in 2010, were examined to identify all those instances where anidulafungin could have been appropriately used, instead of other antifungals.

Based on the analysis of medical records, the substitution index of the other antimycotics with anidulafungin has enabled us to calculate its potential use and the saving that the hospital might be able to achieve.

Results The analysis revealed a frequently inappropriate use of various antifungal drugs.

The review of medical reports confirmed a $70 \%$ substitution index of liposomal amphotericin B with anidulafungin.

In 2010, the hospital used 9,237 vials of caspofungin, anidulafungin and liposomal amphotericin B.

If we assume $100 \%$ use of anidulafungin in instances where it would be appropriate in the two ICUs, the hospital could make savings exceeding Euro 100,000 per year.

Results The ICUs in question account for $18 \%$ of the total vials. The possible savings that could be made by extending the analysis and application of the mathematical model to the entire hospital have not yet been investigated, but the model has confirmed the initial assumption of possibly saving money by using anidulafungin, according to approved indications, in substitution for other antimycotic drugs.

No conflict of interest.

\section{OHP-027 EDUCATIONAL MODEL FOR IT SYSTEMS}

doi:10.1136/ejhpharm-2013-000276.401

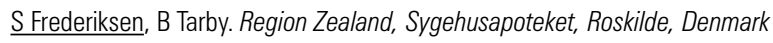

Background An electronic documentation management system had been implemented, replacing and uniting three previously paper-based documentation systems. 130 users located on 6 different locations needed to be educated in the new system.

Purpose To evaluate the training on the new system. Would it be possible for a large number of employees at 6 different locations to use an educational model, called Less Is More (LIM) to rapidly learn a new IT system, with a high degree of satisfied users?

Materials and Methods The training of the personnel was carried out by educating 30 supervisors over a period of 5 days. The supervisors conducted the education of the remaining personnel, based on the principles and materials of LIM. The education model (LIM), is based on simple graphic displays with very simple drawings, that do not require e.g. PowerPoint. The graphic displays give a visual overview of the system. The graphic displays are supplemented by a short storey, telling the procedure of the system. In addition the drawings are followed up by a single page, navigation note with short instructions on how to carry out a specific procedure.

The evaluation was performed using a user survey of the course. The users were to complete a questionnaire, rating relevant statements on a scale from extremely satisfactory, very satisfactory, satisfactory, less than satisfactory and not satisfactory.

Results A survey conducted after the course showed that all 30 supervisors found the method extremely satisfactory, very satisfactory or satisfactory. 100 users on 6 different locations were subsequently educated in a period of 4 weeks, and reports from the system show that the system is being used as desired.

Conclusions We would recommend LIM as an educational model for IT systems, it was used successfully with a high degree of satisfied users.

No conflict of interest.

\section{OHP-028 EFFECT OF A CLOSED SYSTEM DEVICE AND NEW CLEANING PROCEDURE ON SURFACE CONTAMINATION WITH CYTOSTATICS}

doi:10.1136/ejhpharm-2013-000276.402

ZS Órás, I Higyisán, M Kis Szölgyémi. Bajcsy-Zsilinszky Hospital, Pharmacy, Budapest, Hungary

Background The potential for contamination associated with handling cytostatic drugs exists in the workplace despite compliance with the protective measures for the safe handling of cytostatics.

Purpose To investigate the efficacy of using closed system drug transfer devices and implementing a new cleaning procedure for environmental cytostatics contamination in the central cytostatics department.

Materials and Methods Wipe samples were taken from five defined areas in March, 2011: Laminar air flow (LAF) cabinet, workbench, floor in front of the LAF cabinet, transport box and the handle of the refrigerator located in the make-ready room. They were tested for contamination with 8 substances (5-Fluorouracil (5-FU), cyclophosphamide (CP), ifosfamide (Ifos), gemcitabine (Gem), etoposide (Eto), methotrexate (MTX), pacli-taxel (Pac), docetaxel (Doc)) using LC-MS/MS. After seven months the test was repeated on the same surfaces (except the refrigerator handle) after the implementation of PhaSeal closed-system drug transfer device and $0.1 \mathrm{~m} \mathrm{NaOH}$ decontamination solution.

Results In the first test the level of substances wiped from the refrigerator handle was under the detection limit. The LAF cabinet 